\title{
Actualización: \\ El paciente con insuficiencia renal crónica pre-terminal
}

\author{
The patient with pre-terminal chronic kidney disease
}

Alberto Sadler ${ }^{\ddagger}$ y Carlos Musso ${ }^{\S}$

\begin{abstract}
Resumen
La diabetes mellitus, la hipertensión arterial y las glomerulonefritis crónicas son las causas más comunes de insuficiencia renal crónica (IRC) terminal, responsables aproximadamente de 35\%, 30\% y 10\% de los casos, respectivamente. La presencia de un filtrado glomerular menor a $60 \mathrm{~mL} / \mathrm{min} / 1,73 \mathrm{~m} 2$ se asocia a un aumento significativo de riego cardiovascular, hospitalización y mortalidad. Por eso es importante detectar y tratar tempranamente la IRC con el objetivo de enlentecer su progresión.

El tratamiento contra la progresión de la IRC se basa en el tratamiento específico de su etiología, en la implementación de medidas para disminuir la hiperfiltración y en la disminución de los factores de riesgo cardiovascular. Los cuidados de los pacientes en tratamiento con diálisis peritoneal o hemodiálisis son similares a los de un portador de nefropatía crónica más la implementación de una dieta hiperproteica, además de brindarle una adecuada dosis de diálisis y control del acceso dialítico. El transplante renal es el tratamiento principal de la IRC terminal.
\end{abstract}

\begin{abstract}
Diabetes mellitus, hypertension and chronic glomerulonephritis are the most common causes of end stage chronic kidney disease (CKD), responsible for approximately $35 \%, 30 \%$ and $10 \%$ of cases, respectively.

A Glomerular filtration rate below $60 \mathrm{~mL} / \mathrm{min} / 1,73 \mathrm{~m} 2$ is associated with a significant increase in cardiovascular risk, hospitalization and mortality. Therefore it is important to early detect and treat CKD in order to slow its progression.

Management of CKD is based on the specific treatment of its etiology, in implementing measures to reduce hyperfiltration and reduce cardiovascular risk factors. Except for the implementation of a high protein diet and the provision of an adequate dialysis dose and dialysis access control the care of patients on peritoneal dialysis or hemodialysis are similar to those of CKD carriers. Kidney transplantation is the primary treatment of CRF terminal.
\end{abstract}

Palabras clave: insuficiencia renal crónica preterminal, actualización, abordaje, manejo, prevención, accesos vasculares. Key words: chronic kidney disease, treatment, prevention, review, vascular access.

Sadler A, Musso C. El paciente con insuficiencia renal crónica pre-terminal. Evid Act Práct Ambul. Jul-Set 2012;15(3):104-111.

\section{Introducción}

Existe en las sociedades modernas un aumento progresivo de pacientes portadores de insuficiencia renal crónica (IRC), de modo que la prevalencia de esta entidad es actualmente del 11 al $17 \%$ en los países desarrollados ${ }^{1}$. Este incremento en la IRC es el resultado de la conjunción de diversos factores tales como el aumento en la sobrevida de los pacientes portadores de enfermedades cardiovasculares, la epidemia de diabetes mellitus tipo 2, el sedentarismo propio del estilo de vida moderno y el incremento en la expectativa de vida de la población general sumado a los cambios renales propios del envejecimiento ${ }^{2}$.

Desde hace dos décadas existe consenso de que la morbilidad y la mortalidad de los pacientes con insuficiencia renal crónica terminal (IRCT) puede ser mejorada con la derivación temprana de los pacientes a un equipo nefrológico, específicamente para evitar el comienzo catastrófico de la diálisis y asegurar la salud de los pacientes al comenzase los cuidados correspondientes. El fundamento de esta estrategia es que, de no proveerse el adecuado tratamiento a los pacientes con IRC, estos frecuentemente comienzan a dializar enfermos de hipertensión arterial, anemia, hiperparatiroidismo, acidosis metabólica y desnutrición, pudiendo todos estos inconvenientes evitarse o atenuarse mediante un tratamiento adecuado $\mathrm{y}$, frecuentemente, multidisciplinario ${ }^{3}$.

\section{Epidemiología}

Como lo atestiguan los resultados de varios estudios 4,5 , la presencia de un filtrado glomerular menor de $60 \mathrm{~mL} / \mathrm{min} / 1,73 \mathrm{~m}^{2}$ se asocia a un aumento significativo de riego cardiovascular (infarto miocárdico, accidente cerebrovascular), de hospitalización y de mortalidad.

De allí la importancia entonces de detectar y tratar tempranamente la IRC con el objeto no sólo de enlentecer su progresión, sino también de disminuir su riesgo cardiovascular. Es así que la expectativa de vida de un paciente de 49 años con IRCT es de alrededor de siete años**.

Además, el costo de la terapia sustitutiva renal consume una gran parte de los presupuestos sanitarios ${ }^{\text {tt }}$.

Por último, el transplante renal, que constituye el tratamiento de fondo de la IRCT, tiene todavía el problema de que la disponibilidad de órganos es escasa y que las listas de espera son largas ${ }^{\ddagger}$.

\section{Medición de la función renal}

Si bien el criterio estándar para la medición del filtrado glomerular es la depuración de inulina, este estudio es demasiado engorroso como para ser realizado en forma rutinaria. En la práctica clínica se utilizan para el diagnóstico de la disminución de la tasa de filtrado glomerular (TFG) y el seguimiento de la progresión de la enfermedad renal, en orden creciente de prescisión: 1) la determinación de la creatinina plasmática, 2) el aclaramiento de creatinina y el aclaramiento de creatinina con cimetidina ${ }^{\S \S}$

Con respecto a la creatininemia deben tenerse presente que en pacientes con atrofia muscular, ésta puede sobreestimar el filtrado glomerular. Sin embargo, a partir de la creatininemia puede estimarse el filtrado glomerular mediante diversas fór-

¥Servicio de Clínica Médica del Hospital Italiano de Buenos Aires. alberto.sadler@ hospitalitaliano.org.ar

$\S$ Servicio de Nefrología de Hospital Italiano de Buenos Aires. Departamento de Medicina del Instituto Universitario Hospital Italiano de Buenos Aires. carlos.musso @ hospitalitaliano.org.ar

** Dicha expectativa es más de veinte años para la población general de la misma edad y más de ocho, para los individuos con cáncer de colon.

"Por ejemplo en EE.UU. el gasto estimado durante 2010 en terapia sustitutiva renal es de 28.000 millones de dólares, mientras que en Europa el tratamiento dialítico supone "t Por ejemplo en EE.UU. el gasto estimado durante 2010 en terapia sustitutiva renal es de 28 asignación de un $2 \%$ del total del presupuesto sanitario a una población menor al $1 \%$.
la asignas

\# De esta forma, los candidatos ideales para ser transplantados deben, incluso dentro de los programas mundiales más competitivos, aguardar años hasta recibir un órgano. ${ }_{\$ 5}$ Bloquea la secreción tubular de la creatinina haciendo entonces que toda la creatinina urinaria excretada refleje exclusivamente la creatinina filtrada. 
mulas $^{6}$ tales como la de Cockcroft-Gault o la de la modificación de la dieta en pacientes con enfermedad renal (Modification of diet in renal disease: MDRD), que se describen a continuación $^{\star \star *}$ :

\section{Fórmula de Cockcroft - Gault (CG)}

Filtrado glomerular $=(140-$ edad en años $) x$ (peso corporal en $\mathrm{kg}) / 72$ x creatininemia ( $\times 0,85$ en mujeres)

Fórmula de Modificación de la dieta en pacientes con enfermedad renal (MDRD)

Filtrado glomerular $=186 \times$ creatininemia $-1.154 \times$ edad en años $-0.203 \times(0,742$ si es mujer) $\times(1,210$ si es de raza negra)

Otro de los marcadores que puede emplearse para evaluar el filtrado glomerular es la cistatina $\mathrm{C}^{7}$, un polipéptido no glicosilado miembro de la super familia de los inhibidores de la cisteina proteinasa, que se encuentra codificada en un gen presente en la mayor parte de las células nucleadas ${ }^{\dagger t+}$. Su valor sérico normal en jóvenes sanos oscila entre 0,7 y $1,57 \mu \mathrm{g} / \mathrm{mL}$ y se ha demostrado que constituye un marcador de filtrado glomerular superior a la creatininemia. Sin embargo, algunos factores como la edad avanzada, el sobre peso, el género masculino, la alta talla y el tabaquismo pueden elevar sus niveles séricos.

\section{Insuficiencia renal aguda vs. crónica}

Inicialmente, al evaluar un paciente con aumento de la creatinina se debe intentar definir si se trata de un paciente con insuficiencia renal aguda (IRA) o crónica. Los resultados de algunos exámenes de laboratorios pueden aclarar el interrogante en forma inmediata. Si estos no se encuentran disponibles, ciertos datos clínicos, de laboratorio y de estudios por imágenes pueden ser de gran ayuda. Los pacientes con IRA se encuentran generalmente más sintomáticos que los que presentan IRC. Además, es frecuente encontrar un evento clínicamente significativo previo a la elevación de los valores de la urea y la creatinina: hipovolemia, obstrucción urinaria, administración de sustancias nefrotóxicas como los antiinflamatorios no esteroides (AINE), contrastes iodados, etc. Inversamente, en los pacientes con IRC, la anemia, la hipocalcemia y la hiperfosfatemia son habitualmente más significativas que en los pacientes con IRA. La ecografía renal, que evidencia ambos riñones pequeños e hiperecogénicos, es diagnóstica de IRC. Cabe aclarar que tanto la IRC como la IRA pueden cursar con riñones ecográficamente normales, el ejemplo clásico es la IRC secundaria a nefropatía diabética.

\section{Estadíos de la insuficiencia renal crónica}

La Fundación Nacional del Riñón de EE.UU. (en inglés Nacional Kidney Foundation: KDOQI) ha propuesto estratificar a la IRC en cinco estadios desde el 1 (el más leve) hasta el 5 (el más grave), basándose en el nivel del filtrado glomerular ajustado para el área de superficie corporal. Ver cuadro 1.
Cuadro 1: estadíos de la insuficiencia renal crónica propuestos por la Fundación Nacional del Riñón de EE. UU.

\begin{tabular}{c|c|c}
\hline Estadio & Filtrado Glomerular & Prevalencia \\
\hline $\mathbf{1}$ & Mayor a $89 \mathrm{~mL} / \mathrm{min}$ con daño renal & $3,3 \%$ \\
\hline $\mathbf{2}$ & $89 \mathrm{a} 60 \mathrm{~mL} / \mathrm{min}$ con daño renal & $3,0 \%$ \\
\hline $\mathbf{3}$ & 59 a $30 \mathrm{~mL} / \mathrm{min}$ & $4,3 \%$ \\
\hline $\mathbf{4}$ & 29 a $15 \mathrm{~mL} / \mathrm{min}$ & $0,2 \%$ \\
\hline $\mathbf{5}$ & Menor a $15 \mathrm{~mL} / \mathrm{min}$ ó en diálisis & $0,2 \%$ \\
\hline
\end{tabular}

Fuente:

Levey, AS, Coresh, J, Balk, E, Kausz, AT. National Kidney Foundation practice guidelines for chronic kidney disease: evaluation, classification, and stratification. Ann Intern Med 2003; 139:137.

De acuerdo a este consenso, para confirmar el diagnóstico de los pacientes con estadíos iniciales ( 1 y 2 ) en los que el filtrado glomerular está por encima de $60 \mathrm{~mL} / \mathrm{min} / 1,73 \mathrm{~m}^{2}$, es necesario contar además con alguna prueba de daño renal: biopsia renal patológica, sedimento urinario alterado (proteinuria y/o hematuria glomerular), alteraciones séricas secundarias a disfunción renal (hiperkalemia, acidosis metabólica, etc.) y/o alguna alteración renal evidente en estudios por imágenes (ecografía, tomografía computada, y/o resonancia nuclear magnética).

Por el contrario, los estadios más graves de la IRC (estadios 3, 4 y 5) se definen por que el paciente presenta un filtrado glomerular debajo de 60,30 y $15 \mathrm{~mL} / \mathrm{min} / 1,73 \mathrm{~m}^{2}$ respectivamente.

En este punto es importante destacar, que debido a que existe una caída progresiva del filtrado glomerular $(1 \mathrm{~mL} / \mathrm{min}$ año a partir de los 35 años de edad) inherente al envejecimiento renal normal, las personas ancianas (65 a 80 años) y muy ancianas (mayores de 80 años) sanas poseen un filtrado glomerular de alrededor de $70 \mathrm{~mL} / \mathrm{min} / 1,73 \mathrm{~m}^{2}$ y $50 \mathrm{~mL} / \mathrm{min} / 1,73 \mathrm{~m}^{2}$ respectivamente, sin que esto signifique que padezcan IRC, desde el momento que esta hipofiltración senil no se acompaña de anemia, alteración del metabolismo fosfo-cálcico, hematuria glomerular, ni proteinuria significativa (menor a $0,5 \mathrm{~g} / \mathrm{día}$ ). Además, la hipofiltración senil no se traduce en un aumento de la creatininemia debido a la reducción de la masa magra propia del anciano (sarcopenia senil), que es la fuente a partir de la cual se sintetiza la creatinina.

\section{Etiología de la insuficiencia renal crónica}

La diabetes mellitus, la hipertensión arterial y las glomerulonefritis crónicas son las causas más comunes de IRCT, responsables aproximadamente de $35 \%, 30 \%$ y $10 \%$ de los casos, respectivamente. El $25 \%$ restante corresponde a nefropatía obstructiva, infecciones, enfermedad renal poliquística, nefritis intersticial y otras.

En cuanto al estudio inicial de los pacientes con IRC, estos se deben tratar de categorizar en uno de los dos siguientes grupos: 1) enfermedades glomerulares; 2) enfermedades vasculares o intersticiales.

*** Cada una de estas fórmulas posee sus ventajas y desventajas. La ecuación de MDRD predice el filtrado glomerular sin ajustar para la superficie de área corporal, mientras que la fórmula de CG sobrestima el filtrado glomerular en los rangos bajos de filtrado. Por otra parte hay estudios que han documentado en ancianos que el verdadero filtrado glomerular correlaciona mejor con la estimación realizada a través de la fórmula de CG que con la estimada por la MDRD.

t+t Su ritmo de producción parece ser independiente de la masa corporal, y de presencia de enfermedades inflamatorias y/o malignas. La cistatina C filtra libremente a nivel glomerular, para ser luego completamente reabsorbida y metabolizada a nivel tubular proximal. Dado que su concentración urinaria es muy baja, no puede ser utilizada para medir el filtrado glomerular mediante la medición de su aclaramiento plasmático. 


\section{Enfermedades glomerulares}

Las enfermedades glomerulares se caracterizan por la presencia de cilindros eritrocitarios en el sedimento de orina, alto porcentaje de glóbulos rojos dismórficos, proteinuria glomerular y/o evidencia clínica de enfermedad sistémica. Las enfermedades glomerulares pueden ser primarias o secundarias. Son enfermedades glomerulares primarias la glomeruloesclerosis mesangial (tanto IgA como no $\lg A$ ), la glomerulonefritis focal y segmentaria, la nefropatía membranosa, y las glomerulonefritis membranoproliferativa y extracapilar. Son secundarias las nefropatías diabética, lúpica, por amiloidosis. etc. Está generalmente aceptado que el paciente con glomerulopatía no diabética debe ser biopsiado con el fin de obtener un diagnostico etiológico y pronóstico, y consecuentemente realizar un tratamiento específico.

\section{Enfermedades renales vasculares o intersticiales}

Estas enfermedades se caracterizan por la presencia de un sedimento urinario con pocas manisfestaciones patológicas, menos de $3 \mathrm{~g} / 24 \mathrm{~h}$ de proteinuria y por no asociarse a una enfermedad sistémica relacionada a glomerulopatía. Por ejemplo, nefroangioesclerosis hipertensiva, nefropatía por abuso de AINE, nefrolitiasis, nefropatía isquémica, idiopática y/o relacionada con anormalidades anatómicas como la enfermedad renal poliquística y la uropatía obstructiva.

Identificación temprana de los pacientes con insuficiencia renal crónica pre-terminal

El primer paso para mejorar el pronóstico de los pacientes con IRC es su detección precoz.

Una creatinina plasmática mayor o igual a $1,5 \mathrm{mg} / \mathrm{dL}$ en mujeres jóvenes, a $2 \mathrm{mg} / \mathrm{dL}$ en hombres jóvenes o a $1 \mathrm{mg} / \mathrm{dL}$ en ancianos, indica enfermedad renal significativa, lo que implica que ese paciente debería ser sometido a una evaluación exhaustiva.

Se debe intervenir muy precozmente, especialmente en pacientes con condiciones sistémicas como hipertensión arterial, diabetes mellitus, enfermedades inmunológicas (ej. lupus eritematoso sistémico o vasculitis) infecciones urinarias a repetición, uropatía obstructiva, nefrolitiasis, infección por el virus de la inmunodeficiencia humana (VIH), o con enfermedades renales como las mencionadas más arriba.

Conocer estos factores de riesgo es de fundamental importancia para adoptar medidas con el fin de retardar la progresión de la caída del filtrado glomerular.

Los pacientes diabéticos con proteinuria, definidos como proteinuria cualitativa de "dos cruces" o " $2+$ " en exámenes de orina tomados en dos diferentes oportunidades o con proteinuria cuantitativa mayor o igual a $200 \mathrm{mg} / 24 \mathrm{~h}$ pueden ser considerados como portadores de nefropatía diabética y casi con seguridad progresarán a IRCT.

En los individuos con diabetes mellitus la microalbuminuria que no es detectada por el examen con tira reactiva en el examen de orina habitual- es un marcador temprano de nefropatía y su presencia (30 a $300 \mathrm{mg} / \mathrm{día}$ ) indica la necesidad de realizar un tratamiento agresivo para prevenir la progresión de la enfermedad renal.

Es por eso que los pacientes con diabetes mellitus sin macroproteinuria en el examen de orina y sin caída de filtrado deben ser rastreados anualmente en búsqueda de microalbuminuria desde los cinco años del diagnóstico en los pacientes con diabetes tipo $1 \mathrm{y}$ desde el momento del diagnóstico en los pacientes con diabetes tipo $2^{10}$.

Vale destacar además que la concurrencia de hipertensión arterial en los pacientes con diabetes mellitus con proteinuria es altamente predictiva de progresión de caída del filtrado glomerular, lo que implica un probable desarrollo de IRCT en un lapso de meses a pocos años.

La presencia de proteinuria en los pacientes VIH positivos virtualmente define nefropatía por $\mathrm{VIH}$; pudiendo ser muy rápido el curso hacia el desarrollo de IRCT (semanas o meses).

Manejo del paciente con insuficiencia renal crónica preterminal

Estrategias para retardar la progresión hacia la insuficiencia renal crónica terminal

El tratamiento contra la progresión de la IRC se basa, por un lado, en el tratamiento específico correspondiente a su etiología ${ }^{11}$, y por otro, al tratamiento de los factores de riesgo asociados con la enfermedad cardiovascular: hipertensión arterial, tabaquismo, hiperglucemia, hiperlipidemia, anemia, hiperfosfatemia y proteinuria, así como el uso de fármacos cardioprotectores.

\section{Control de la hipertensión arterial}

La hipertensión arterial (HTA) es una manifestación frecuente en los pacientes con IRC, afectando hasta el $85 \%$ de esta población. Es nociva porque produce nefro-angioesclerosis y porque, independientemente de su etiología, acelera la progresión de la IRC. Su efecto deletéreo estaría dado por que produce hipertrofia y/o hipertensión intraglomerular con la consecuente glomeruloesclerosis y caída del filtrado glomerular.

Existe actualmente bastante consenso en que la tensión arterial en los pacientes con IRC debe mantenerse por debajo de $130 / 80 \mathrm{mmHg}^{9}$ y de que cualquier antihipertensivo que logre controlar la tensión arterial retardaría la progresión de la enfermedad renal . Por otro lado, en los pacientes con diabetes mellitus el control adecuado de la tensión arterial lentifica la progresión de la enfermedad renal, independientemente del control de la glucemia.

Con la progresión de la enfermedad renal disminuye la capacidad de los riñones de mantener el balance de sodio. Por lo tanto, una dieta hiposódica y el tratamiento con diuréticos pueden ser necesarios para mantener un balance adecuado de agua y sodio, ya que, además, el control de la TA es virtualmente imposible en los pacientes con sobrecarga de volumen. Por otro lado, la combinación de HTA y sobrecarga de volumen incrementa el trabajo cardiaco provocando hipertrofia ventricular izquierda, que constituye un factor de riesgo mayor para enfermedad coronaria, insuficiencia cardiaca y muerte súbita. Actualmente se cree que la progresión hacia la IRCT es el resultado de la hiperfiltración, la hipertensión glomerular y la proteinuria. Los inhibidores de la enzima convertidora de la angiotensina (IECA) y los bloqueantes de los receptores de angiotensina II (BRA II) han demostrado ser eficaces en reducir la presión capilar glomerular y la hiperperfusión, así como también en reducir la proteinuria y, con esto, retardar la progresión de cualquier enfermedad renal ${ }^{13,14}$, en especial la nefropatía dia- 
bética $^{\ddagger \neq \ddagger}$

La asociación con diuréticos del asa es de suma utilidad para manejar la HTA en los pacientes con IRC pre-terminal, dado que, como veremos más adelante, estos se encuentran frecuentemente hipervolémicos ${ }^{\S \S}$. Los bloqueantes de los canales de calcio no dihidropiridínicos (diltiazem y verapamilo) tienen efecto antiproteinúrico y pueden utilizarse como drogas de segunda línea en el tratamiento de la HTA en pacientes con IRC.

\section{Abandono del tabaquismo}

El tabaquismo es un factor de riesgo tradicional asociado a enfermedad cardiovascular y estudios recientes sugieren que fumar acelera considerablemente la progresión de la enfermedad renal. Además, es importante que los fumadores dejen de fumar ya que dicha progresión se atenúa ${ }^{15}$.

\section{Uso de fármacos nefroprotectores}

Esta familia está integrada por los inhibidores de la enzima convertidora de angiotensiona (IECA), los bloqueantes del receptor de antiotensina II (BRA II), la espironolactona y los inhibidores de la renina ${ }^{* \star * *}$.

La administración de IECA disminuye el riesgo relativo de progresión de la IRC hasta un $50 \%$, según los resultados de varios estudios prospectivos y multicéntricos ${ }^{16}$. Recordamos que los IECA y los BRA II están contraindicados en mujeres embarazadas y en pacientes con el antecedente de angioedema.

Un aspecto importante a remarcar es que el efecto renoprotector de los IECA se asocia claramente al efecto antiproteinúrico. Vale decir que en quienes se observa un descenso significativo de la proteinuria se puede anticipar un efecto favorable en la estabilización de la función renal o al menos un enlentecimiento en la progresión de la caída del filtrado glomerulartttt. Lo mencionado explica el concepto de que Ios IECA o BRA II deben utilizarse en cualquier paciente con IRC, sobre todo si presenta proteinuria, independientemente de su presión arterial. El objetivo buscado es conseguir, de ser posible, una proteinuria menor a $0,2 \mathrm{~g} / \mathrm{día}$.

La utilidad de estos fármacos es mayor si se administran precozmente, en lo posible en la fase proteinúrica y cuando la función renal todavía está normal. En general se prefiere comenzar con un IECA y no con un BRA II, dado el mayor número de estudios que avalan sus beneficios favorables en la progresión de la IRC y su menor costo. Algunos estudios recientes sugieren la utilización de la combinación sinérgica de IECA con BRA II para pacientes que no logran los objetivos deseados, lo que potenciaría sus efectos antiproteinúricos, bloqueando a distintos niveles la acción de la angiotensina II¥¥¥ .

\section{Restricción de las proteínas de la dieta}

Si bien la evidencia es limitada, la restricción de las proteínas de la dieta también ha demostrado mejorar la hemodinamia glomerular, reducir la proteinuria y, con esto, retardar la progresión de la enfermedad renal, especialmente en personas con diabetes.

Actualmente se recomienda una dieta con 0,8 g/ $\mathrm{kg} / \mathrm{día}$ de proteínas en pacientes con IRC pre-terminal, manteniendo una ingesta de calorías no menor a $35 \mathrm{kcal} / \mathrm{kg} / \mathrm{día}$. Sin embargo esta restricción proteica está contraindicada durante los cuadros clínicos de estrés significativo, en pacientes con enfermedades severas concomitantes y/o con mala nutrición.

Para los pacientes con IRC moderada (tasa de filtrado glomerular entre 25 y $55 \mathrm{~mL} / \mathrm{min} / 1,73 \mathrm{~m} 2$ de superficie corporal) el Grupo de Trabajo del Instituto Nacional de Salud de EE.UU. recomienda una ingesta proteica estándar (mayor a 0,8g/kg por día) ${ }^{8}$.

Dado que la desnutrición proteica es un marcador independiente de mortalidad en pacientes con IRCT, se recomienda que estos pacientes sean seguidos conjuntamente con nutricionistas entrenados.

\section{Control de la glucosa plasmática}

La nefropatía diabética se produce en $35 \%$ de los pacientes con diabetes tipo 1 y aproximadamente en $15 \%$ de los de tipo 2. En los pacientes diabéticos que cursan con microalbuminuria o nefropatía diabética se ha demostrado que además de controlar estrictamente la tensión arterial, administrar IECA o BRA II y reducir las proteínas de la dieta, el control estricto de la glucemia también retarda francamente la progresión de la enfermedad renal. Por lo tanto, se recomienda mantener la hemoglobina glicosilada por debajo de $7 \%$ y también mantener la glucemia preprandial entre 70 y $120 \mathrm{mg} \%$, recomendándose interconsultar al especialista en quienes resulta difícil el manejo de la glucemia ${ }^{14}$.

\section{Dislipidemia}

Valores séricos elevados de colesterol total, LDL y triglicéridos son factores de riesgo tradicionales de la enfermedad cardiovascular. También hay información proveniente de estudios animales que sugieren que la hiperlipidemia podría causar daño glomerular por depósito a nivel mesangial. Por las razones antes expuestas el uso de hipolipemiantes: estatinas y fibratos podría contribuir a reducir la progresión de la enfermedad renal y el riesgo cardiovascular ${ }^{18}$. La dosis de algunas estatinas (rosuvastatina) debe ajustarse en el contexto de insuficiencia renal severa o en combinación con fibratos,

\footnotetext{
\#\# Estos fármacos también inhiben el proceso de esclerosis glomerular, inflamación intersticial y fibrosis. Por esto, los IECA y los BRA II son los fármacos de elección a la hora de comenzar el tratamiento de la HTA en este grupo de pacientes.

${ }_{9 \varsigma \S}$ Frecuentemente se comete el error de no utilizar dosis lo suficientemente altas de diuréticos del asa. En ocasiones y para lograr el efecto diurético deseado, esa dosis tiene que ser repetida durante el resto del día. Recordamos además que es recomendable no emplear diuréticos tiazídicos en pacientes con creatininemia mayor a 1,8 mg/dL o filtrado glomerular menor de $40 \mathrm{~mL} / \mathrm{min} / 1,73 \mathrm{~m} 2$, con la única excepción de algunos diuréticos símil-tiazídicos como la metolazona, que pueden utilizarse en pacientes con insuficiencia renal severa.

**** La angiotensina II interviene en forma significativa en la progresión de la IRC y los IECA y los BRA II han demostrado disminuir significativamente la progresión de la IRC, tanto en animales como en seres humanos.

Antes de conocerse su influencia protectora sobre la evolución de la IRC, se observó que los IECA poseían un efecto antiproteinúrico, tanto en individuos con nefropatías diaAntes de conocerse su influencia protectora sobre la evolución de la IRC, se observó que los IECA poseían un efecto antiproteinúrico, tanto en individuos con nefropatías dia-
béticas como en no diabéticas. Además de sus acciones hemodinámicas y antihipertensivas, los IECA ejercen un efecto sobre la pared glomerular, restaurando su capacidad de filtro para las macromoléculas. El efecto antiproteinúrico se observa con todos los IECA, se produce rápidamente en días o semanas y es en gran medida independiente de su efecto hipotensor.

trtt Por el contrario, aquellos pacientes que no evidencian reducción de la proteinuria continúan evolucionando con el mismo ritmo de progresión que antes de recibir el IECA. Las propiedades profibrogénicas de la proteinuria a nivel tubulointersticial pueden explicar esta estrecha correlación entre el descenso de la proteinuria y el efecto favorable de los IECA en la progresión de la IRC.

\#\#¥ Si pese a la combinación de IECA y BRA II no se logra reducir significativamente la proteinuria puede intentarse lograrlo sumando al esquema anterior espironolactona o un inhibidor de la renina (aliskiren), con el correspondiente monitoreo de la tensión arterial y de la kalemia, dado el riesgo potencial de hipotensión y/o hiperkalemia que esta combinación conlleva.
} 
recomendándose apuntar en esta población a valores de menores de $200 \mathrm{mg} / \mathrm{dL}$ de colesterol total, menores de $100 \mathrm{mg} / \mathrm{dL}$ de colesterol LDL y menores de $200 \mathrm{mg} / \mathrm{dL}$ de triglicéridos.

\section{$\underline{\text { Anemia }}$}

Si bien la anemia asociada a la insuficiencia renal crónica es generalmente normocítica y normocrómica (a raíz de una menor producción de eritropoyetina secundaria a la disminución de la masa renal funcionante), puede sin embargo ser de tipo multifactorial, por ejemplo por disminución de la vida media de los eritrocitos, por hemólisis, por ferropenia (pérdidas gastrointestinales y extracciones para estudios de laboratorio). Además, el eritrón puede estar inhibido parcialmente por enfermedades concurrentes y sus tratamientos específicos como infecciones, síndrome de la inmunodeficiencia adquirida (SIDA), malnutrición, y por el efecto inespecífico de las enfermedades crónicas ${ }^{\S \S \S}$.

Se recomienda iniciar la terapéutica con eritropoyetina cuando la hemoglobina disminuye de $10 \mathrm{~g} / \mathrm{dL}$, y una vez descartada o resuelta la presencia de ferropenia documentando que el paciente tenga una ferritina mayor a $300 \mathrm{ng} / \mathrm{mL}$ y una saturación de transferrina mayor a $25 \%$. De requerirse reposición de hierro, la ferroterapia intravenosa (hierro sacarato) ha sido más efectiva que la oral en la corrección de la anemia en esta población.

En pacientes tratados con eritropoyetina se busca llegar a una hemoglobinemia entre 11 y $12 \mathrm{~g} / \mathrm{dL}$, ya que se ha documentado que hemoglobinas superiores a $13 \mathrm{~g} / \mathrm{dL}$ no confieren beneficio y se asocian a un aumento del riesgo cardiovascular ${ }^{19}$.

\section{Metabolismo fosafo-cálcico}

El desajuste del metabolismo fosfo-cálcico podría estar asociado con el progreso de la enfermedad cardiovascular (hipertrofia ventricular izquierda y calcificaciones vasculares y valvulares cardíacas), y también con el progreso de la enfermedad renal. Por otra parte, la hiperfosfatemia no tratada estimula el hiperparatiroidismo secundario y la osteodistrofia renal, a lo que se suma el hecho del efecto que la paratohormona es por sí misma una de las principales toxinas urémicas ${ }^{20}$.

El manejo de la hiperfosfatemia se basa en una dieta restringida en alimentos muy ricos en fósforo (productos lácteos, bebidas colas, y carnes procesadas), con el objetivo de que el consumo diario de fósforo ronde entre los 800 y los $1000 \mathrm{mg}$. En el estadio 4 de la IRC se apunta a mantener un rango de fosfatemia de 2,7 a $4,6 \mathrm{mg} / \mathrm{dL}$ y en el estadío 5 , un rango de 3,5 a $5,5 \mathrm{mg} / \mathrm{dL}$.

De no ser suficiente esta estrategia, debe sumarse al tratamiento, el uso de quelantes del fósforo. El quelante más recomendable es el carbonato de calcio, tomado con las comidas. Además del carbonato de calcio, existen otros quelantes del fósforo como el sevelamer y el carbonato de lantano. Entre los quelantes del fósforo conviene evitar el hidróxido de aluminio, que puede producir toxicidad alumínica y el citrato de calcio, que incrementa la absorción intestinal de aluminio.

Asimismo, es prudente restringir la ingesta de calcio total en pacientes con IRC a 1500 a 2000 mg/día a fin de minimizar el riesgo de calcificaciones vasculares, apuntando a lograr un valor del producto fosfo-cálcico (calcemia $\mathrm{x}$ fosfatemia) menor a $55 \mathrm{mg} 2 / \mathrm{dL}^{2}$.

Cuando la TFG disminuye por debajo de 40 a $70 \mathrm{~mL} / \mathrm{min}$, la PTH comienza a incrementarse. El tratamiento en esta situación está basado en la restricción dietética de fósforo, en el uso de quelantes del mismo y en el uso de 1,25 dihidroxivitamina D (calcitriol), supresor de la liberación de PTH. Los niveles de vitamina $D$ suelen ser bajos en los pacientes con IRC, no sólo por su reducida síntesis renal, sino además por su escaso ingreso dietario y exposición solar. El aporte de calcitriol aumenta los niveles de calcio en la sangre e incrementa la absorción intestinal fósforo. Es por esta razón que NO se debe administrar vitamina $D$ hasta que la hiperfosfatemia no esté controlada, y sólo cuando el calcio sérico corregido sea mayor a $9,5 \mathrm{mg} / \mathrm{dL}$.

Con respecto a la vitamina $25-\mathrm{D}$, se recomienda que sus valores séricos sean de al menos $30 \mathrm{ng} / \mathrm{mL}$, ya que niveles inferiores se asocian a grave debilidad muscular. Si no pudiera llegarse a dichos niveles existe consenso en aportarle al paciente suplementos de ergocalciferol. Otra alternativa para el tratamiento farmacológico del hiperparatiroidismo es el cinacalcet, un calcimimético que aumenta la sensibilidad del receptor del calcio hacia este catión divalente en la glándula paratiroidea, lo que se traduce en una disminución en la secreción de paratohormona. A diferencia del calcitriol, que está contraindicado en el contexto de hipercalcemia y/o hiperfosfatemia por aumentar la absorción intestinal de estas sustancias y poder empeorar en consecuencia el producto-fosfo-cálcico, el cinacalcet puede emplearse en este escenario.

\section{Nutrición}

Los pacientes desnutridos al inicio de la diálisis presentan una mortalidad mayor que los pacientes que no lo están ${ }^{\star \star \star \star *}$. Al comienzo de la diálisis, la mayoría de los pacientes están mal nutridos y con una albúmina sérica de alrededor de $3,2 \mathrm{~g} / \mathrm{dL}$, mientras que el promedio habitual de los pacientes que se encuentran en diálisis es algo mejor (3,75 g/dL) apuntándose en esta última población a una albuminemia deseable de 4 $\mathrm{g} / \mathrm{dL}$, ya que valores por debajo de este umbral se asocian a una mayor mortalidad. Por lo tanto, la pérdida de peso y, específicamente, el descenso de la albúminemia por debajo de $3,5 \mathrm{~g} / \mathrm{dL}$, indican desnutrición, que debe ser corregida mediante dieta y/o diálisis ${ }^{17+t+t t}$.

Si bien existe controversia respecto de si la restricción proteica enlentezca el progreso de la enfermedad renal, se recomienda una ingesta proteica de $0,8 \mathrm{~g} / \mathrm{kg} /$ día durante la etapa pre-dialítica. Por el contrario, durante la etapa dialítica (sobre todo en pacientes que realizan diálisis peritoneal) se debe incrementar dicha ingesta proteica.

Respecto de la obesidad, en pacientes no urémicos se asocia con una mayor mortalidad, con un incremento del riesgo cardiovascular y con deterioro renal (hiperfiltración glomerular); mientras que en pacientes en diálisis, parece no tener asociación con la mortalidad.

Es prudente, además, recomendar a los pacientes con IRC preT la reducción del contenido de potasio de la dieta. En estos

\$s\$s Si bien todavía no queda claro, hay información que sugiere que la corrección temprana de la anemia prevendría el desarrollo de hipertrofia ventricular izquierda en pacientes con IRC. Además, el tratamiento de la anemia con eritropoyetina podría disminuir el ritmo de progresión de la IRC, y existe evidencia de que la corrección de la anemia mejora la capacidad cognitiva en pacientes ancianos con IRC

${ }_{\star \star \star \star \star \star}$ La prueba de referencia para la evaluación del estado nutricional en esta población es la evaluación clínica del estado nutricional llamada evaluación global subjetiva (en inglés: global subjective assessment o GSA).

$\mathrm{t+t+1}$ No obstante, debe destacarse que la albuminemia puede estar descendida como consecuencia de un estado inflamatorio a modo de reactante inverso de fase aguda y que esta posibilidad debe ser explorada antes de afirmar que simplemente, el paciente está desnutrido. 
pacientes, Ios IECA y los BRA II deben ser administrados con suma cautela, así como los diuréticos ahorradores de potasio. Recordamos que los diuréticos del asa ayudan a prevenir la hiperpotasemia y en pacientes seleccionados, la hiperpotasemia debe ser controlada mediante bisacodilo o resinas de intercambio catiónico, que aumentan la excreción fecal de potasio.

La acidosis metabólica requiere especial atención debido a sus consecuencias metabólicas y, además, porque puede producir síntomas idénticos a los de la uremia: anorexia, astenia y disnea. La principal consecuencia de la acidosis metabólica es que promueve el catabolismo proteico y, por lo tanto, la desnutrición. Puede ser prevenida con la administración de tabletas o soluciones de bicarbonato de sodio. El objetivo es mantener el bicarbonato sérico en alrededor de $22 \mathrm{mmol} / \mathrm{L}$. Habitualmente, se comienza el tratamiento con una dosis de bicarbonato de 0,5 a $1 \mathrm{meq} / \mathrm{kg} / \mathrm{día}$, aumentandose la dosis paulatinamente hasta lograrse los valores mencionados. A su vez, la sobrecarga de sodio inducida por la administración de bicarbonato debe ser manejada intensificando el tratamiento diurético para evitar la sobrecarga de volumen.

El cuadro 2 resume los principales lineamiento relativos a la nutrición del paciente con IRC preT.

Cuadro 2: dieta recomendada para pacientes con insuficiencia renal crónica pre-terminal.

\begin{tabular}{|c|c|c|}
\hline \multicolumn{2}{|l|}{ Proteínas } & $0,8 \mathrm{~g} / \mathrm{kg} / \mathrm{dí} a$ \\
\hline \multicolumn{2}{|l|}{ Energía } & Más de 35kcal/día (excepto en obesos) \\
\hline \multirow{3}{*}{$\begin{array}{c}\text { Distribución } \\
\text { calórica de la } \\
\text { dieta }\end{array}$} & Grasa & 30 a $40 \%$ \\
\hline & Hidratos de carbono & 50 a $60 \%$ \\
\hline & \begin{tabular}{|l|} 
Proteínas \\
\end{tabular} & 8 a $10 \%$ \\
\hline \multicolumn{2}{|l|}{ Sodio } & 1000 a 3000mg/día \\
\hline \multicolumn{2}{|l|}{ Potasio } & 40 a $70 \mathrm{meq} /$ día \\
\hline \multicolumn{2}{|l|}{ Fósforo } & 5 a $10 \mathrm{mg} / \mathrm{kg} / \mathrm{día}$ \\
\hline \multicolumn{2}{|l|}{ Calcio } & 1400 a $1600 \mathrm{mg} / \mathrm{dí}$ \\
\hline \multicolumn{2}{|l|}{ Hierro } & Mayor a 10mg/día \\
\hline
\end{tabular}

Estrategias farmacológicas cardioprotectoras

Los pacientes portadores de IRC (especialmente los que están en estadios 3 a 5) tienen mayor riesgo de desarrollar enfermedad cardiovascular, por lo que se benefician de una estrategia cardioprotectora que incluye el uso de beta bloqueantes (sobre todo aquellos que no se acumulan en el contexto de caída del filtrado glomerular como propanolol y carvedilol) y de ácido acetilsalicílico (100 mg/día).

Detección y tratamiento de los factores que disminuyen reversiblemente el filtrado glomerular

\section{Insuficiencia cardíaca}

El bajo volumen minuto cardiaco reduce significativamente el flujo plasmático renal y la tasa de filtrado glomerular, mientras que la activación del sistema renina-angiotensina-aldosterona y el sistema nervioso simpático producen vasoconstricción intrarrenal con la consecuente disminución del filtrado glomerular. Los pacientes con IRC presentan dificultad para excretar sodio y agua. Si a esto se le agrega la incrementada reabsorción de sodio en el nefrón distal, se comprende que la sobrecarga de volumen y las complicaciones como el edema agudo de pulmón sean frecuentes.

Como describimos previamente, el tratamiento se realiza, fun- damentalmente, con diuréticos del asa como furosemida, dado que los diuréticos tiazídicos están contraindicados en pacientes con IRC con filtrado glomerular inferior a $40 \mathrm{~mL} / \mathrm{min} / 1.73 \mathrm{~m}^{2 \neq \neq \neq ł \neq}$. Los IECA y los BRA II, ampliamente utilizados en forma exitosa en pacientes con insuficiencia cardiaca sistólica y diastólica deberán ser administrados con cautela. Se debe monitorear los valores de urea, creatinina y potasio para evitar un empeoramiento severo de la función renal y/o hiperkalemia.

\section{Depleción de volumen}

La depleción de volumen puede disminuir la perfusión renal y, por lo tanto, disminuir el filtrado glomerular. Evitar este estado en pacientes con IRC es de capital importancia y debe ser corregido en forma inmediata.

\section{Obstrucción del tracto urinario}

Mediante ecografía puede detectarse fácilmente la presencia de uropatía obstructiva. La causa más común, especialmente en individuos de edad avanzada, es la hipertrofia prostática. Otras causas son la litiasis, el cáncer de próstata, la necrosis papilar renal, otras malignidades pelvianas, los trastornos neurogénicos de la vejiga y la fibrosis retroperitoneal. Estas comorbilidades deben ser corregidas precozmente por el urólogo.

\section{Drogas}

Antinflamatorios no esteroides (AINE): los AINE inhiben la síntesis de las prostaglandinas vasodilatadoras renales y como consecuencia, producen una vasoconstricción que se asocia a una disminución del filtrado glomerular. Los pacientes con IRC son extremadamente sensibles a este mecanismo, especialmente en presencia de hipovolemia, utilización de diuréticos, insuficiencia cardiaca y edad avanzada. Además de esta IRA vasomotora reversible, los AINE pueden producir nefritis intersticial, síndrome nefrótico (cambios mínimos), hiperkalemia y retención hidrosalina. Por lo tanto, en lo posible debe ser evitada la administración de AINE en los pacientes con IRC y en caso de ser utilizados, la urea y la creatinina deben ser monitoreadas estrictamente.

Otras drogas nefrotóxicas y ajuste de las dosis a la función renal del paciente: los aminoglucósidos son una causa frecuente de IRA e IRC. Estos antibióticos deben tratar de evitarse en pacientes con insuficiencia renal. En caso de ser necesarios, se debe ajustar su dosis al filrado glomerular y/o medir los niveles plasmáticos de los mismos. Existe una muy numerosa lista de drogas nefrotóxicas o que requieren adecuar su dosis a la función renal, de modo que debe siempre tenerse esto presente antes de medicar a un paciente portador de insuficiencia renal.

\section{Medios de contraste radiológicos}

Los contrastes iodados utilizados en los estudios radiológicos pueden desencadenar IRA o agravar una IRC preexistente ${ }^{\S \S s \S}$. Existen fundamentalmente dos teorías que intentan explicar la fisiopatología de la nefropatía por contraste: 1) vasoconstricción renal mediada por alteraciones en el óxido nítrico y endotelina y; 2) injuria directa por el radiocontraste y probable generación de radicales libres del oxígeno (explicaría los efectos beneficiosos de la acetilcisteina como antioxidante en la prevención de la injuria renal por contrastes iodados).

Son factores de riesgo para el desarrollo de insuficiencia renal por contraste la IRC previa, la deshidratación, la nefropatía diabética con insuficiencia renal, el mieloma múltiple, la enfermedad renal por cadenas livianas, la insuficiencia cardíaca severa, y la cantidad total y el tipo de medio de contraste utilizado.

\footnotetext{
\#\#\# De requerirse en este contexto el empleo de un diurético para combinar con furosemida (bloqueo bitubular), puede emplearse la metolazona.

${ }_{\$ \$ \$ \varsigma \S}$ Cuando un paciente desarrolla toxicidad renal por medios de contraste, sus valores de creatinina comienzan a ascender en 24 ó 48 horas, hacen su pico alrededor de la semana y regresan a los valores basales entre los 10 y los 14 días.
} 
Algunos pacientes con elevación de la creatinina requieren diálisis transitoriamente, quedando unos pocos con necesidad permanente de la misma.

En los pacientes con IRC los contrastes iodados deben evitarse siempre que sea posible. En caso de estricta necesidad, se recomienda utilizar productos no iónicos y que sean iso o hiposmolares, que son menos dañinos para el riñón.

La profilaxis con solución salina intravenosa (IV) al 0,9\%, a razón de $1 \mathrm{~mL} / \mathrm{kg} / \mathrm{hora}$, desde 12 horas antes hasta 12 horas después del procedimiento disminuye significativamente la incidencia y la severidad de la insuficiencia renal por contraste en los pacientes con IRC previai ${ }^{21,22 .}$

La administración de acetilcisteina a una dosis de 600mg cada 12 horas por vía oral durante el día previo y durante el día de la administración de contraste (un total de cuatro dosis), conjuntamente con solución salina IV, ha demostrado ${ }^{23}$ ser más eficaz en reducir la incidencia de nefropatía por contraste en comparación con la administración de solución salina solamente. Cuando se administran medios de contraste iodado en pacientes con factores de riesgo deben suspenderse en lo posible los AINE, Ios IECA y los BRA II con el fin de permitirle al riñón una mejor adaptación hemodinámica intra-renal, y por ende, una disminución de la probabilidad de que se instale una insuficiencia renal aguda.

Vale destacar que no existe evidencia que avale la utilización de medidas profilácticas en pacientes con creatinina normal y ausencia de los factores de riesgo mencionados anteriormente. En los últimos tiempos se han comenzado a realizar angiografías con gadolinio, pero este contrate debe tratar de evitarse en pacientes con filtrado glomerular inferior a 60 $\mathrm{mL} / \mathrm{min} / 1,73 \mathrm{~m}^{2}$, debido que se asocia al desarrollo de fibrosis sistémica nefrogénica.

\section{Estenosis renovascular}

Debido a que una adecuada revascularización por diferentes métodos puede resolver este problema, la estenosis renovascular debe ser sospechada en los pacientes que se presentan con un incremento dramático del valor de creatinina con la utilización de IECA o BRA II, hipertensión arterial refractaria con insuficiencia renal progresiva, insuficiencia renal inexplicable por otra causa en ancianos con enfermedad vascular, asimetría renal significativa (mayor a un centímetro) en la ecografía, ó bien la triada clínica de HTA refractaria, edema de pulmón recurrente e insuficiencia renal.

\section{Manejo del paciente con insuficiencia renal crónica terminal}

Actualmente existe consenso ${ }^{24}$ respecto de algunos "indicadores esenciales" de la calidad del manejo de pacientes con insuficiencia renal crónica terminal (IRCT) que tienen que ver con una diálisis adecuada (control de la uremia como un proxi del estado en que se encuentran resto de las toxinas urémicas) y al igual que en la etapa predialítica, el control de la hipertensión arterial, el tabaquismo, la anemia, la dislipidemia, el metabolismo fosfocálcico, la glucemia y la nutrición.

Al comienzo de la diálisis, aproximadamente $25 \%$ de los pacientes tiene un acceso vascular permanente para la diálisis, muchos no han tenido un adecuado seguimiento de su IRCpreT por un nefrólogo y sólo una proporción muy pequeña ha sido manejada por un equipo multidisciplinario.

Existe evidencia de que una derivación oportuna al nefrólogo (por lo menos 60 días antes del comienzo de la diálisis) resulta en internaciones más cortas para la iniciación de la diálisis, menos días de internación durante el año subsiguiente (13 vs.
23 días) y una mortalidad significativamente menor durante el primer año de diálisis (22 vs. $41 \%)^{25}$. Una vez que la diálisis se vuelve estable, la salud de los pacientes mejora drásticamente como consecuencia del logro de los objetivos de los indicadores esenciales. Sin embargo resaltamos que es de buena práctica no esperar hasta el comienzo de la diálisis para controlar efectivamente los indicadores antes mencionados.

\section{Acceso para la diálisis}

La derivación tardía de los pacientes con IRC preT a un equipo nefrológico limita la elección del tipo de diálisis, lo mismo que el acceso para la misma. Los problemas con los accesos para la diálisis constituyen la causa principal de morbilidad y hospitalización en los pacientes con IRCT, particularmente en el período inicial del tratamiento de reemplazo de la función renal ${ }^{26}$.

Para la hemodiálisis se prefiere como acceso la fístula arteriovenosa por su menor incidencia de complicaciones y su mayor durabilidad. Se recomienda confeccionarla cuando la TFG se encuentra alrededor de los $20 \mathrm{~mL} / \mathrm{min} / 1,73 \mathrm{~m}^{2}$. También se puede crear una anastomosis implantando un conducto artificial de polietileno (o góretex) entre una arteria y una vena. Desafortunadamente, estos se complican muy frecuentemente, desarrollándose estenosis venosa en la proximidad del sitio anastomótico, con la consecuente reducción de la sobrevida funcional del implante, lo que requiere la colocación de catéteres para hemodiálisis, procedimientos de desobstrucción de los implantes o la confección de una nueva anastomosis. Estos inconvenientes, además del consumo progresivo de las estructuras vasculares nativas para la colocación de dichos implantes, puede amenazar la sobrevida a largo plazo de los pacientes y encarecer significativamente el tratamiento.

Cabe mencionar que en EE.UU. sólo 20 a 25\% de los pacientes utiliza fístulas arteriovenosas como acceso vascular. Esto resulta difícil de comprender, dado que la mayoría tiene arterias y venas braquiales adecuadas para la confección de una fístula nativa (arteriovenosa). Una razón para explicar este fenómeno podría ser la derivación tardía a un equipo nefrológi$\mathrm{co}$, ya que el desarrollo de una fístula arteriovenosa requiere por lo menos seis a ocho semanas antes de ser utilizada, mientras que un implante puede ser usado dentro de los tres a los 14 días de su colocación.

La tercera opción dentro de los accesos vasculares es un catéter percutáneo en una vena central. Estos se infectan frecuentemente y causan sepsis potencialmente fatales. También predisponen a la trombosis de la vena en la que están colocados. Estos catéteres deben ser, en lo posible, transitorios mientras se consigue una mejor opción. Por lo tanto, los catéteres centrales como acceso permanente solo deben indicarse en caso de imposibilidad total de obtener un acceso braquial ya que la trombosis de una vena subclavia descarta el brazo homolateral como sitio probable de accesos vasculares. Los catéteres femorales no deben ser utilizados como accesos permanentes por la elevada incidencia de infecciones relacionadas a los mismos. La preservación de las venas braquiales de los pacientes con IRC preT debe tenerse muy presente. Se deben evitar o limitar las venopunturas proximales a la muñeca, dado que estas podrían dañar sitios adecuados para accesos vasculares. Los pacientes ancianos, diabéticos, obesos y aquellos que recibieron corticoterapia en forma prolongada, tienen frecuentemente malos lechos vasculares para la construcción de un acceso vascular. Algunos de estos pacientes pueden entrar en un programa de ejercicios para desarrollar los vasos sanguíneos braquiales y con esto mejorar el índice de éxito en cuanto a la confección de una fístula adecuada.

Con respecto a la diálisis peritoneal, que a diferencia de la 
hemodiálisis es una modalidad autodialítica, se recomienda que el paciente realice el curso de entrenamiento (dos clases) cuando su TFG es de $20 \mathrm{~mL} / \mathrm{min} / 1,73 \mathrm{~m} 2$ y que recién se le coloque el catéter peritoneal cuando la TFG sea de 10 $\mathrm{mLmin} / 1,73 \mathrm{~m} 2$, ya que éste puede utilizarse a partir del mismo día de su colocación. Dado el carácter domiciliario, continuo, cerrado (no exposición de la sangre), y preservador de la diuresis residual (cuya conservación mejora la sobrevida), es recomendable que el paciente comience, de ser posible, su tratamiento dialítico mediante la modalidad peritoneal. Asimismo, dado que la membrana peritoneal (filtro) se agota tras varios años de uso, si para dicho momento el paciente no ha sido aun transplantado, puede continuar dializando mediante hemodiálisis con la ventaja de haber preservado sus venas durante la etapa peritoneal y tenerlas disponibles en consecuencia para la construcción del acceso vascular.

\section{Modalidades terapéuticas de la insuficiencia renal crónica terminal}

La derivación temprana a un equipo nefrológico puede ser de gran utilidad a la hora de elegir el tratamiento de la IRCT. Las modalidades posibles son esencialmente tres: 1) hemodiálisis, 2) diálisis peritoneal, tanto en su modalidad manual: diálisis peritoneal contínua ambulatoria (DPCA) como en su modalidad automatizada (APD), 3) transplante renal, el cual constituye el tratamiento principal de la IRCT, desde el momento que cualquiera de las modalidades dialíticas antes mencionadas tan sólo le brinda al paciente una función renal equivalente a un TFG de $9 \mathrm{~mL} / \mathrm{min} / 1,73 \mathrm{~m}^{2}$, a diferencia del transplante renal que le otorga una función renal plena. La decisión entre estos tres métodos de tratamiento depende de numerosos factores: características clínicas del paciente (enfermedades comórbidas, posibilidades de accesos vasculares, edad, etc.), tipo de modalidad terapéutica disponible en el centro asistencial en cuestión, disponibilidad de órganos y preferencias del paciente.

\section{Comienzo de la diálisis}

Las indicaciones de diálisis de urgencia están ampliamente aceptadas y son las siguientes: uremia sintomática (nauseas, vómitos), encefalopatía, pericarditis urémica, sobrecarga de volumen (edema pulmonar), hiponatremia, hiperkalemia y/o acidosis metabólica que no responden al tratamiento médico.

La indicación electiva de diálisis varía según algunas características de los pacientes, especialmente si presentan desnutrición. El comienzo de la diálisis se recomienda cuando la TFG se acerca a $10 \mathrm{~mL} / \mathrm{min} / 1,73 \mathrm{~m}^{2}$. Es importante remarcar que debido a que la creatinina se filtra a nivel glomerular y también se secreta a nivel del túbulo proximal, y que dicha secreción se incrementa progresivamente a medida que cae el filtrado glomerular, resulta entonces que la depuración de la creatinina sobrestima el filtrado glomerular, y más aun en el contexto de una nefropatía crónica. Por este motivo un marcador práctico y confiable del filtrado glomerular, es la depuración o "clearance" minutado de creatinina con bloqueo de la secreción de creatinina mediante el uso de cimetidina.

\section{Tratamientos no dialíticos de la insuficiencia renal crónica severa}

Existen grupos que han propuesto manejos alternativos de la uremia y eventual retraso del ingreso a diálisis en pacientes muy ancianos mediante diversas estrategias. Es así que una de ellas está basada en la prescripción de una dieta ultrabaja en proteínas (0,3 g/k/día) suplementada con aminoácidos esenciales y análogos cetósicos de los aminoácidos. Otra de estas estrategias propone el uso de carbón activado por vía oral como una forma de remover toxinas urémicas por vía intestinal ${ }^{27}$. Finalmente, otros grupos están investigando la potencial utilidad del suministro oral de bacterias consumidoras de nitrógeno, propias de la flora colónica habitual ${ }^{28}$ (probióticos), como otra estrategia de manejo intestinal del síndrome urémico.

Referencias

1. Gorriz Teruel, Martin de Francisco AL. Epidemiología e impacto sociosanitario de la IRC. En Hernando Avendaño L (Ed). Nefrología Clínica. Madrid. Panamericana. 2009: 857-862. 2. Sarnak, MJ, Levey, AS, Schoolwerth, AC, Coresh, J. Kidney disease as a risk factor for development of cardiovascular disease: a statement from the American Heart Association Councils

on Kidney in Cardiovascular Disease, High Blood Pressure Research, Clinical Cardiology, and Epidemiology and Prevention. Circulation 2003; $108: 2154$.
3 . Levey, AS, Coresh, J, Balk, E, Kausz, AT. National Kidney Foundation practice guidelines for chronic kidney disease: evaluation, classification, and stratification. Ann Intern Med 2003 139:137.

4. Hansson L, Zanchetti A, Carruthers SG, et al. Effects of intensive blood-pressure lowering and low-dose aspirin in patients with hypertension: principal results of the Hypertension Optimal Treatment (HOT) randomised trial. HOT Study Group. Lancet. 1998;351:1755-1762.

5. VALsartan In Acute myocardial iNfarcTion (VALIANT) trial: baseline characteristics in context. Eur J Heart Fail. 2003:5:537-544.

6. Teruel J y col. La ecuación de Cockroft-Gault es preferible a la ecuación MDRD para medir el filtrado glomerular en la insuficiencia renal crónica avanzada. NEFROLOGíA. Volumen 27. Número 3. 2007.

7. Roos J F, Doust J, Tett S E, Kirkpatrick C M. Diagnostic accuracy of cystatin C compared to serum creatinine for the estimation of renal dysfunction in adults and children: a meta-analysis. Clinical Biochemistry 2007; 40(5-6): 383-391.

8. Levey, AS, Coresh, J, Balk, E, Kausz, AT. National Kidney Foundation practice guidelines for chronic kidney disease: evaluation, classification, and stratification. Ann Intern Med 2003; 139:137.

9. Musso CG, Macías Nuñez JF, Oreopoulos DG. Physiological similarities and differences between renal aging and chronic renal disease. J Nephrol. 2007; 20(5):586-7.

10. Guía de Práctica Clínica Nacional sobre Prevención, Diagnóstico y Tratamiento de la Diabetes Mellitus Tipo 2 Para el Primer Nivel de Atención. Ministerio de Salud de la Nación. 2009. Disponible en http://www.msal.gov.ar

11. Ministerio de Salud de la Nación, Argentina. Guía de Práctica Clínica sobre Prevención y Detección Precoz de la Enfermedad Renal Crónica en Adultos en el Primer Nivel de Atención. 2010.

Disponible en URL: http://msal.gov.ar/ent/SRV/Guias/PDF/2GPC\%20de\%20prevencion\%20de\%20enfermedad\%20renal\%20para\%20el\%20PNA\%20Marzo\%202010.pdf (último acceso 28/09/12)

12. LV J, Neal B, Ehteshami P, Ninomiya T, Woodward M, et al. (2012) Effects of Intensive Blood Pressure Lowering on Cardiovascular and Renal Outcomes: A Systematic Review and MetaAnalysis. PLoS Med 9(8): e1001293. doi:10.1371/journal.pmed.1001293

13. Jafar, TH, Schmid, CH, Landa, M, et al. Angiotensin-converting enzyme inhibitors and progression of nondiabetic renal disease. A meta-analysis of patient-level data. Ann Intern Med 2001;

135:73.
14. Hilgers, KF, Mann, JF. ACE Inhibitors versus AT(1) Receptor Antagonists in Patients with Chronic Renal Disease. J Am Soc Nephrol $2002 ; 13: 1100$.

15. Scotish Intercolegiate Guideline Network (SIGN). Diagnosis and management of chronic kidney disease. A National Clinical Guideline. 2008.

16. Canadian Society of Nephrology.Guidelines for the management of chronic kidney disease. 2008

17. Praga Terente M. Tratamiento conservador de la insuficiencia renal crónica. Medidas generales y manejo dietético. En Hernando Avendaño L (Ed). Nefrología Clínica. Madrid. Panamericana. 2009: 863-869.

18. CARI Guidelines.Caring for Australasians with Renal Impairment.2008.

19. Ajay K. Singh, M.B., B.S., Lynda Szczech, M.D., Kezhen L. Tang, Ph.D., Huiman Barnhart, Ph.D., Shelly Sapp, M.S., Marsha Wolfson, M.D. and Donal Reddan, M.B., B.S. for the CHOIR Investigators Correction of Anemia with Epoetin Alfa in Chronic Kidney Disease. N Engl J Med 2006; 355:2085-2098November 16, 2006DOI: 10.1056/NEJMoa065485

20. $\mathrm{Hsu} \mathrm{CH}$. Calcium and phosphate metabolism management in chronic renal disease. Michigan. Springer. 2006

21. Mueller, C, Buerkle, G, Buettner, HJ, et al. Prevention of contrast media-associated nephropathy: randomized comparison of 2 hydration regimens in 1620 patients undergoing coronary

angioplasty. Arch Intern Med 2002; 162:329.

22. Trivedi, HS, Moore, H, Nasr, S, Aggarwal, K. A randomized prospective trial to assess the role of saline hydration on the development of contrast nephrotoxicity. Nephron 2003; 93:C29. 23. Tepel, M, Van Der Giet, M, Schwarzfeld, C, et al. Prevention of radiographic-contrast-agent-induced reduction in renal function by acetylcysteine. N Engl J Med 2000; $343: 180$.

25. Bradbury JD Dialysis Handbook. Philadelphia. Kluwer. 2008 , Pisoni RL, Port FK, Gillespie BW. Predictors of early mortality among incident US hemodialysis patients in the Dialysis Outcomes and Practic terns Study (DOPPS). Clin J Am Soc Nephrol 2007; 2: 89-99.

26. Schwab SJ. Hemodialysis vascular access: the Achilles' heel remains. Kidney Int 2007; 72: 665-6.

27. Musso CG, Michelangelo H, Reynaldi J, Martinez B, Vidal F, Quevedo M, Parot M, Waisman G, Algranati L Combination of oral activated charcoal plus low protein diet as a new alternative for handling in the old end-stage renal disease patients. Saudi J Kidney Dis Transpl. 2010; $21(1): 102-4$

28. Ranganathan N, Friedman E, Tam P, Rao V, Ranganathan P, Dheer R. Probiotic dietary supplementation in patients with stage 3 and 4 chronic kidney disease: a 6 month pilot scale trial in Canada. Current Medical Research and opinion. 2009; 25(8): 1919-30 